\title{
Magnetic anisotropy and quantized spin waves in hematite nanoparticles
}

Klausen, Stine Nyborg; Lefmann, Kim; Lindgård, Per-Anker; Kuhn, Luise Theil; Bahl, Christian Robert Haffenden; Frandsen, Cathrine; Mørup, Steen; Roessli, B.; Cavadini, N.; Niedermayer, C.

Published in:

Physical Review B Condensed Matter

Link to article, DOI:

10.1103/PhysRevB.70.214411

Publication date:

2004

Document Version

Publisher's PDF, also known as Version of record

Link back to DTU Orbit

Citation (APA):

Klausen, S. N., Lefmann, K., Lindgård, P-A., Kuhn, L. T., Bahl, C. R. H., Frandsen, C., Mørup, S., Roessli, B., Cavadini, N., \& Niedermayer, C. (2004). Magnetic anisotropy and quantized spin waves in hematite nanoparticles. Physical Review B Condensed Matter, 70(21), 214411.

https://doi.org/10.1103/PhysRevB.70.214411

\section{General rights}

Copyright and moral rights for the publications made accessible in the public portal are retained by the authors and/or other copyright owners and it is a condition of accessing publications that users recognise and abide by the legal requirements associated with these rights.

- Users may download and print one copy of any publication from the public portal for the purpose of private study or research.

- You may not further distribute the material or use it for any profit-making activity or commercial gain

- You may freely distribute the URL identifying the publication in the public portal 


\title{
Magnetic anisotropy and quantized spin waves in hematite nanoparticles
}

\author{
S. N. Klausen,* K. Lefmann, ${ }^{\dagger}$ P.-A. Lindgård, L. Theil Kuhn, and C. R. H. Bahl \\ Materials Research Department, Ris $\varnothing$ National Laboratory, DK-4000 Roskilde, Denmark \\ C. Frandsen and S. Mørup \\ Department of Physics, Technical University of Denmark, DK-2800 Kgs. Lyngby, Denmark \\ B. Roessli, N. Cavadini, and C. Niedermayer \\ Laboratory for Neutron Scattering, Paul Scherrer Institute, CH-5232 Villigen PSI, Switzerland
}

(Received 7 July 2004; published 9 December 2004)

\begin{abstract}
We report on the observation of high-frequency collective magnetic excitations, $\hbar \omega \approx 1.1 \mathrm{meV}$, in hematite $\left(\alpha-\mathrm{Fe}_{2} \mathrm{O}_{3}\right)$ nanoparticles. The neutron scattering experiments include measurements at temperatures in the range $6-300 \mathrm{~K}$ and applied fields up to $7.5 \mathrm{~T}$ as well as polarization analysis. We give an explanation for the field- and temperature dependence of the excitations, which are found to have strongly elliptical out-of-plane precession. The frequency of the excitations gives information on the magnetic anisotropy constants in the system. We have in this way determined the temperature dependence of the magnetic anisotropy, which is strongly related to the suppression of the Morin transition in nanoparticles of hematite. Further, the localization of the signal in both energy and momentum transfer brings evidence for finite-size quantization of spin waves in the system.
\end{abstract}

DOI: $10.1103 /$ PhysRevB.70.214411

PACS number(s): 75.75.+a, 25.40.Fq, 75.30.Ds

\section{INTRODUCTION}

The magnetic dynamics of nanoparticles is an interesting field both technologically and scientifically. As the size of a particle decreases and enters the nanometer scale, the magnetic properties change and new phenomena appear. One example is superparamagnetic relaxation, in which the direction of the total magnetic moment of the particle performs a spontaneous reversal. A related example is collective magnetic excitations, which can be described as a spin wave with wave vector $q=0$ : the individual magnetic moments move coherently and the total (sublattice) spin performs a precession in an effective crystal anisotropy field. This corresponds to the lowest magnetic excitation of nanoparticles, in which the spin waves are predicted ${ }^{1}$ to be quantized with discrete, sharp energy levels, while showing a broadening with respect to the spin wave vector, $q$.

Few experimental studies have been performed on finitesized systems in order to investigate spin wave quantization. Clear evidence for standing spin waves has been found by Brillouin light scattering in ferromagnetic NiFe wires of 1.8 $\mu \mathrm{m}$ width $^{2,3}$ and by pulsed-field techniques in square $\mathrm{NiFe}$ dots of $50 \mu \mathrm{m}$ size. ${ }^{4}$ In magnetic clusters, discrete energy levels have been observed by neutron scattering in $\mathrm{Mn}_{12}$ clusters, ${ }^{5,6}$ and in $\mathrm{Fe}_{8}$ clusters. ${ }^{7}$ In both cluster studies, the reorientation of the total cluster spin was seen, corresponding to collective magnetic excitations, while in $\mathrm{Mn}_{12}$ transitions between different cluster spin values were also observed. This can be seen as a precursor to finite-size quantization of spin waves in nanoparticles, which has not yet been observed.

The magnetic dynamics of nanoparticles of the canted antiferromagnet hematite $\left(\alpha-\mathrm{Fe}_{2} \mathrm{O}_{3}\right)$ is particularly interesting due to a complicated magnetic structure, which gives rise to rich dynamics. ${ }^{8-10}$ Further, an unexplained suppression of the
Morin transition has been observed in hematite nanoparticles. ${ }^{11,12}$ The Morin transition is related to the magnetic anisotropy, and thus studies of the suppression could yield important information on one of the key properties of magnetic nanoparticles.

Superparamagnetic relaxation and low-frequency collective magnetic excitations were directly observed by inelastic neutron scattering in a powder sample of diameter $d=16 \mathrm{~nm}$ hematite particles. ${ }^{9}$ The measurements were performed at a triple-axis spectrometer with a momentum transfer corresponding to the antiferromagnetic (003) reflection (hexagonal indexing), $\boldsymbol{\tau}_{003}=1.37 \AA^{-1}$, and a maximum energy transfer of $1.0 \mathrm{meV}$. The collective magnetic excitations (at $\varepsilon= \pm 0.26 \mathrm{meV}, q=0$ ) were found to be strongly broadened, ${ }^{9}$ to overlap at $\varepsilon=0$. The broadening could be caused by damping, distribution of anisotropy constants, or both. Later high-resolution neutron studies were focused on the study of superparamagnetic relaxation. These measurements were performed ${ }^{13}$ with energy transfers up to 0.75 meV.

In this paper we present inelastic neutron scattering measurements on the same powder sample, as in Refs. 9 and 13, measured with momentum transfers corresponding to the antiferromagnetic (101) reflection, $\tau_{101}=1.51 \AA^{-1}$. We show the existence of a second magnetic excitation mode with $q$ $=0$ and with a resonance frequency of $\sim 1.1 \mathrm{meV}$. The highfrequency mode is seen not only to be reasonably well defined in momentum $(q)$, but also to be clearly localized in energy $(\varepsilon)$. We argue that this implies a discrete spin wave spectrum with an anisotropy gap, in agreement with the theoretical predictions. The measurements allow an estimation of the temperature dependence of the anisotropy connected to the suppression of the Morin transition. 


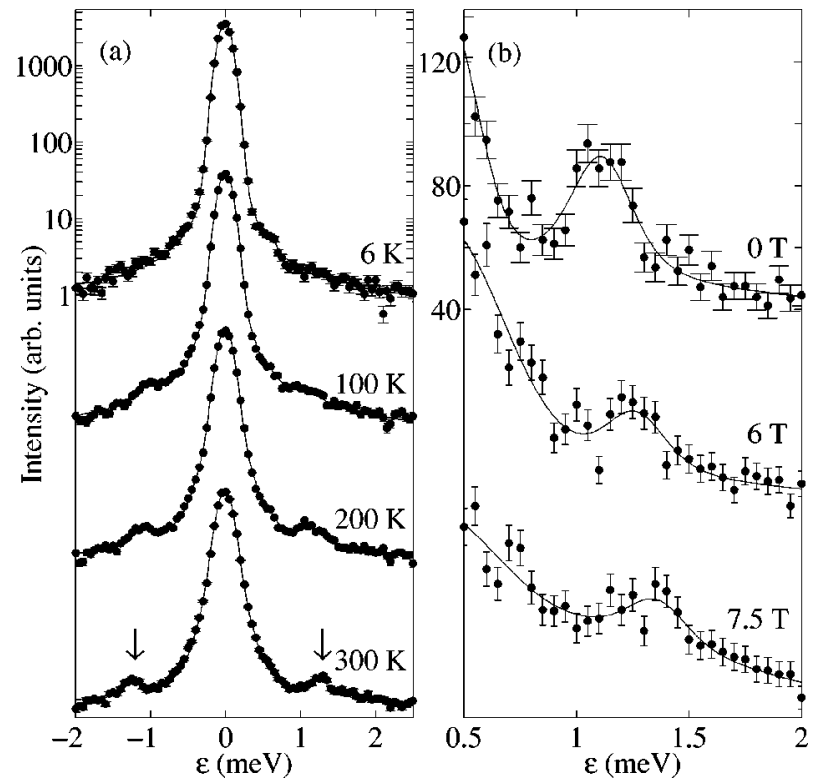

FIG. 1. Inelastic neutron scattering data measured at $\kappa=1.50 \AA^{-1}$, close to $\tau_{101}$. (a) Logarithmic plot of the data at zero applied field at the indicated temperatures. The arrows indicate the position of the high-frequency collective magnetic excitation. (b) Linear plot of the data in applied fields at $T=200 \mathrm{~K}$. The data are displaced by (a) a factor of 100 and (b) a constant. The solid lines represent fits as described in the text. The feature at $0.6 \mathrm{meV}$ in (a) is a background signal as discussed in the text.

\section{THE HEMATITE SAMPLE}

Hematite has the corundum crystal structure, while the magnetic structure is essentially hexagonal with alternating planes of spins along the hexagonal $c$ axis, i.e., along the [001] direction. In bulk hematite at high temperatures, the spins are lying in the basal (001) plane, but below the Morin temperature, $T_{M}=263 \mathrm{~K}$, a spin flop transition takes place to leave the spins parallel to [001]. In nanoparticles below $\approx 20 \mathrm{~nm}$, this Morin transition is suppressed, and only the high-temperature phase is found. In this phase, the spins have a small canting (in bulk $\sim 0.1^{\circ}$ ) away from antiferromagnetic alignment, resulting in a small net magnetization within the plane. Hematite is the most stable form of iron oxide at ambient conditions and is found in vast quantities on Earth, and recently also on Mars. ${ }^{14}$

The powder sample of nanocrystalline hematite is the same as was used in the previous studies. It was prepared by thermal decomposition of $\mathrm{Fe}\left(\mathrm{NO}_{3}\right)_{3} \cdot 9 \mathrm{H}_{2} \mathrm{O}$, as described in Refs. 9 and 10. Mössbauer spectroscopy, electron microscopy, and x-ray diffraction have shown that the sample consists of nanocrystallites of hematite with a mean size of 16 $\mathrm{nm}$ and a small amount of impurities in the form of ferrihydrite, and that the Morin transition is suppressed in the nanoparticles..$^{9,10}$

\section{NEUTRON SCATTERING EXPERIMENTS}

The neutron scattering experiments were performed at the RITA-2 and TASP spectrometers at SINQ, Paul Scherrer In- stitute, Switzerland. The RITA-2 spectrometer was run in the monochromatic point-to-point focusing ${ }^{15}$ and in the monochromatic imaging ${ }^{16}$ analyzer modes. The experiments at TASP were performed in standard triple-axis geometry. Some of the TASP experiments were performed with polarization analysis. Here, the small adjustable guide field at the sample position ensured that the neutron polarization would always point along the scattering vector, $\boldsymbol{\kappa}$. All experiments were performed with constant final neutron energy of 5.0 $\mathrm{meV}$ using relatively loose collimations.

\section{A. Experiments with unpolarized neutrons}

Figure 1 shows the normalized measured scattering intensity versus neutron energy transfer, $\varepsilon=\hbar \omega$, for scans performed at constant neutron momentum transfer $\kappa=1.50 \AA^{-1}$ in zero applied field at the indicated temperatures and at 200 $\mathrm{K}$ in applied fields. In Fig. 1, a peak at $\sim 1.1-1.3 \mathrm{meV}$ is seen at both positive and negative energy transfers. The peak position increases with increasing temperature and increasing field. As we shall discuss later, we ascribe this signal to a high-energy mode of collective magnetic excitations.

The data have been fitted to an elastic signal from incoherent scattering and two damped harmonic oscillator models for the dynamics of the low-frequency and highfrequency precession states. ${ }^{17}$ [Broadening of the elastic signal due to superparamagnetic relaxation can be neglected with the present energy resolution of approximately $100 \mu \mathrm{eV}$ (HWHM); see Ref. 13.] Before correcting for experimental resolution and background, the model is given by

$$
I(\varepsilon)=A_{\mathrm{inc}} \delta(\varepsilon)+\sum_{ \pm} A^{ \pm} \frac{D(\varepsilon)}{\pi} \frac{2 \gamma^{ \pm} \varepsilon_{0}^{ \pm 2}}{\left(\varepsilon^{2}-\varepsilon_{0}^{ \pm 2}\right)^{2}+4 \gamma^{ \pm 2} \varepsilon^{2}},
$$

where $A_{\text {inc }}$ and $A^{ \pm}$are the integrated intensities (areas) of the incoherent elastic signal and the low-frequency (+) and highfrequency (-) inelastic components, respectively. $\gamma^{ \pm}$is the HWHM and $\varepsilon_{0}^{ \pm}$the positions of the inelastic peaks, respectively. $D(\varepsilon)=\varepsilon[n(\varepsilon)+1]$ is the detailed balance factor, where $n(\varepsilon)$ is the Bose factor.

The inelastic background is modeled by a broad Lorentzian signal, found from measurements at other $\kappa$ values. Further, a single Gaussian models an anomalous peak at $\sim 0.6 \mathrm{meV}$, which is a subtle artifact of the open geometry of the RITA-2 spectrometer combined with a nonideal mosaic nature of the PG (002) analyzer crystal. ${ }^{18}$ The energy resolution function used for the temperature series was measured on the hematite sample at $T=6 \mathrm{~K}$. For the field series, it was measured in zero field at $T=200 \mathrm{~K}$. The main component of the resolution function in the two data series is a Gaussian line with HWHM 107(2) $\mu \mathrm{eV}$. Additionally, there is a much weaker Lorentzian component to the resolution function of HWHM 90(25) $\mu \mathrm{eV}$. The parameters $\varepsilon_{0}^{+}$and $\gamma^{+}$of the inelastic component modeling the low-frequency collective magnetic excitations are fixed at the values obtained in the highresolution experiment of Ref. 13. In Fig. 2 the position $\varepsilon_{0}^{-}$ and the area $A^{-}$of the high-frequency peak as obtained from the fits are shown as a function of sample temperature and applied field. 

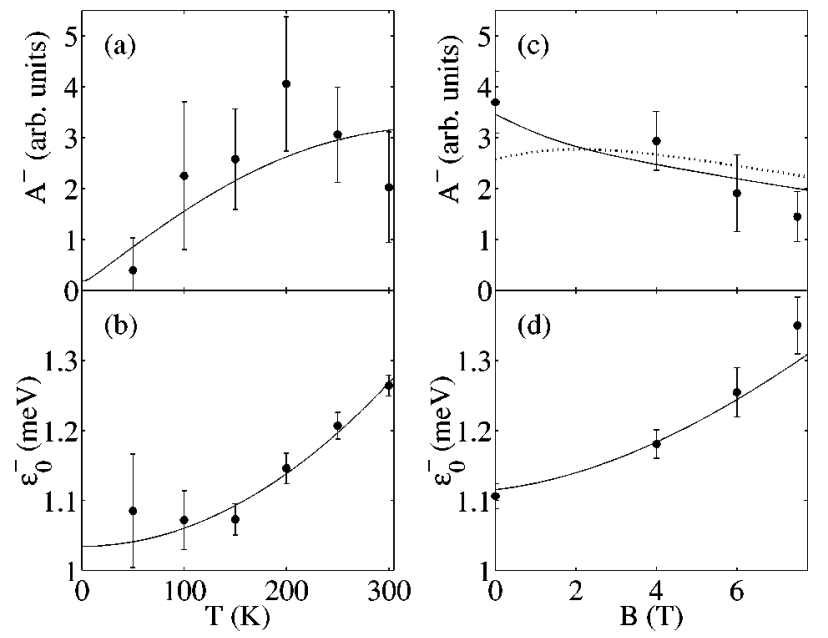

FIG. 2. (a) Area $\left(A^{-}\right)$and (b) position $\left(\varepsilon_{0}^{-}\right)$of the inelastic peak as a function of temperature in zero field. (c) Area $\left(A^{-}\right)$and (d) position $\left(\varepsilon_{0}^{-}\right)$of the inelastic peak as a function of applied field at $200 \mathrm{~K}$. The lines show fits as described in the text.

Figure 3(a) shows diffraction data measured at $200 \mathrm{~K}$. The peaks at $1.372(2) \AA^{-1}$ and $1.517(3) \AA^{-1}$ are the antiferromagnetic (003) and (101) reflections from hematite, respectively. The peak at $1.704(5) \AA^{-1}$ is the structural $(10 \overline{2})$ reflection. In Fig. 3(b) a $\kappa$ scan measured at constant neutron energy transfer $\varepsilon=1.1 \mathrm{meV}$ is shown. A peak at $\sim 1.52 \AA^{-1}$ is clearly seen. The peak at $\sim 1.37 \AA^{-1}$ is mostly due to a tail from the low-frequency excitation, as we will discuss below.

Figure 4 shows a $(\kappa, \omega)$ color map of the inelastic scattering around the magnetic scattering vectors $\boldsymbol{\tau}_{003}$ and $\boldsymbol{\tau}_{101}$ in zero field and $T=200 \mathrm{~K}$. The data were taken with the monochromatic imaging mode, yielding 7 data points per setting. The complete map was taken with 9 energy scans. The method, including data treatment procedure, is detailed in Ref. 18. The statistics of the data in the color map is inferior to the data of Figs. 1 and 3, but the overall tendencies can be more easily seen in a color map. The mode at $\varepsilon=1.1 \mathrm{meV}$ is clearly seen at $\kappa=\tau_{101}$, and a faint signal at the same energy is seen at $\kappa=\boldsymbol{\tau}_{003}$. The (101) mode is clearly localized in $(\kappa, \varepsilon)$ as a relatively sharp peak. This is in contrast to the low-frequency (003) mode, which is broad and has intensity even at $\varepsilon=0$.

\section{B. Experiments with polarized neutrons}

The purely structural reflection at $\kappa=1.70 \AA^{-1}$ has supposedly only non-spin-flip scattering. In the inset of Fig. $3(c)$, we show the polarization analysis of this reflection. From the small value of the measured spin-flip scattering, we determine the overall polarization of the experimental setup, which is comprised of initial polarization, depolarization in the setup, and polarization efficiency of the benders. The value was $P=0.69(9)$.

The main part of Fig. 3(c) shows the results of a polarization analysis of the inelastic peak measured at $\kappa=1.50 \AA^{-1}$, close to $\boldsymbol{\tau}_{101}$. Since the neutron polarization is parallel to $\boldsymbol{\kappa}$, all magnetic scattering is ideally spin-flip scattering. With the
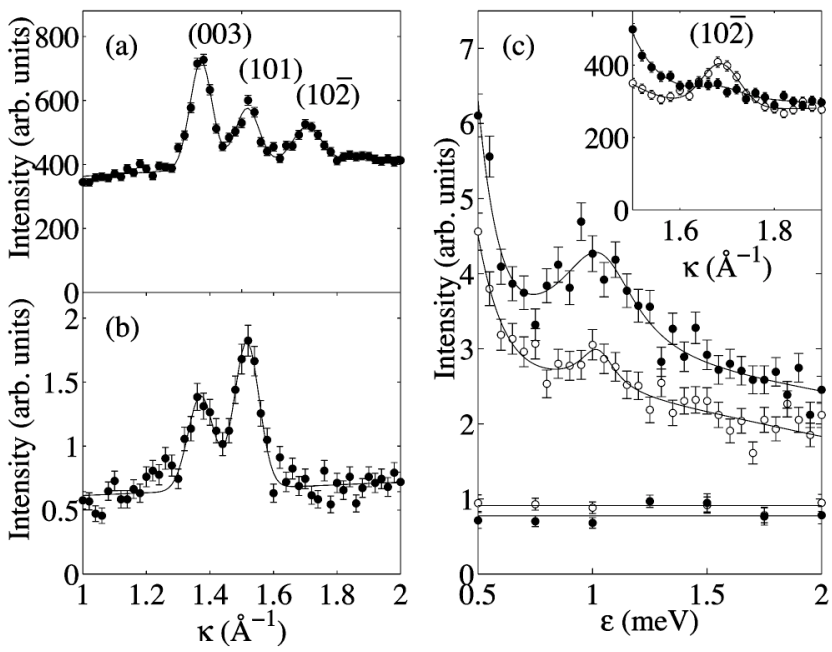

FIG. 3. (a) Diffraction data on hematite at $200 \mathrm{~K}$. (b) Constant$\varepsilon$-scan measured at $\varepsilon=1.1 \mathrm{meV}$ at $200 \mathrm{~K}$. The solid lines are fits to the peaks multiplied with the Debye-Waller factor plus a sloping background. (c) Polarization data on hematite measured at $200 \mathrm{~K}$ in the non-spin-flip channel $(\bigcirc)$ and the spin-flip channel $(\bullet)$. The straight lines represent fits as described in the text. The main panel shows the inelastic intensity measured at the antiferromagnetic reflection at $\kappa=1.50 \AA^{-1}$. The lower straight lines are the corresponding analyzer-turned background in the two channels. The inset shows diffraction data.

present value (and uncertainty) of $P$, this gives that the ratio of the observed ratio of non-spin-flip scattering to spin-flip scattering is expected to be 1:5(2). Indeed, the largest part of the peak at $\sim 1.1 \mathrm{meV}$ is observed in the spin-flip channel. The non-spin-flip to spin-flip fraction is found to be 1:3.1(6). This does not contradict the hypothesis of a purely magnetic origin of the signal.

The background exceeding the analyzer-turned background originates from the sample. Since the intensity in the two channels is equal, except for a factor of 1.44(5), this

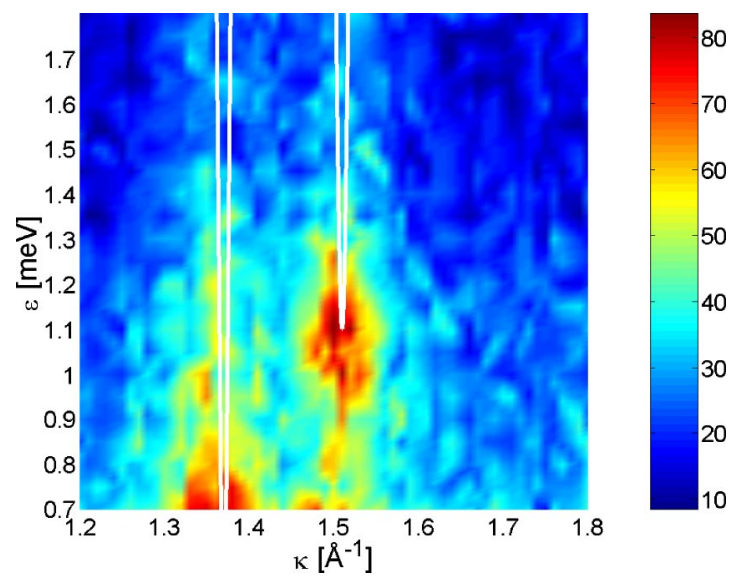

FIG. 4. (Color online) The scattered inelastic intensity at $200 \mathrm{~K}$ and zero field as a function of scattering vector, $\kappa$ (horizontal) and energy transfer, $\hbar \omega$ (vertical). The bar to the right represents the color scale. The solid lines show the dispersion relation of bulk hematite with anisotropy gaps of 1.1 and $0.26 \mathrm{meV}$, as discussed in the text. The data have been normalized as described in Ref. 18. 
signal probably comes from nuclear incoherent scattering, probably from ${ }^{1} \mathrm{H}$. This ratio would be $1: 2$ for nuclear incoherent scattering with a totally polarized beam, ${ }^{19}$ but with the present value of $P$, this would become 1:1.6(1), in agreement with measurements.

\section{DISCUSSION}

\section{A. Quantization of spin waves}

The measured spin wave dispersion relation of bulk hematite is very steep, ${ }^{20}$ with an energy given by $\varepsilon_{q}=\sqrt{c^{2} q^{2}+\varepsilon_{0}^{ \pm 2}}$, where the effective spin wave velocity is $c=200 \mathrm{meV} \AA$. The first excited state with $q \neq 0$ of a spherical nanoparticle has a (standing) wavelength ${ }^{1}$ of $\approx 1.42 d$. For a $d=16 \mathrm{~nm}$ particle (corresponding to $\sim 10^{5}$ spins) this gives the smallest nonzero $q$ value of $q_{\min } \approx 0.028 \AA^{-1}$, corresponding to a minimum energy of $\varepsilon_{\min } \approx 5.6 \mathrm{meV}$ for $\varepsilon_{0}^{ \pm} \leqslant 1 \mathrm{meV}$. The observed signals at $\sim 0.26 \mathrm{meV}$ and $\sim 1.1 \mathrm{meV}$ therefore cannot be caused by finite-size quantization. They must be assigned to uniform magnetic excitations ( $q=0$ spin waves) and they determine the value of the small anisotropy gap, $\varepsilon_{0}^{ \pm}$. This corresponds to the lowfrequency and high-frequency modes of the weak ferromagnetic phase in bulk hematite. 8,20

For spin waves in a bulk isotropic antiferromagnet, the scattering cross section for creation or annihilation of an excitation is proportional to ${ }^{17,19,21}$

$$
\begin{aligned}
I(\varepsilon, \boldsymbol{\kappa}) \propto & \mathcal{J}_{0}\langle S\rangle \sum_{\beta}\left[1-\left(\hat{\boldsymbol{\kappa}} \cdot \hat{\boldsymbol{M}}_{\beta}\right)^{2}\right] \frac{n\left(\varepsilon_{q, \boldsymbol{B}}^{ \pm}\right)+\frac{1}{2} \pm \frac{1}{2}}{\varepsilon_{q, \boldsymbol{B}}^{ \pm}} \\
& \times \delta(\boldsymbol{\kappa} \mp \boldsymbol{q}-\boldsymbol{\tau}) \delta\left(\varepsilon_{q, \boldsymbol{B}}^{ \pm} \mp \varepsilon\right),
\end{aligned}
$$

where $\beta$ is a Cartesian coordinate, and $\hat{\boldsymbol{M}}_{\beta}$ and $\hat{\boldsymbol{\kappa}}$ are unit vectors pointing in one direction of the spin wave amplitude and the scattering vector, respectively. For isotropic spin waves, the sum is conventionally replaced by $1+\left(\hat{\boldsymbol{\kappa}} \cdot \hat{\boldsymbol{M}}_{z}\right)^{2}$, where $z$ is the direction of the ordered moment.

In a powder sample like ours, the orientation of $\tau_{101}$ (the relevant reciprocal lattice vector) is arbitrary. For a given length of the spin wave vector, $q$, the scattering vector, $\boldsymbol{\kappa}$, can thus fall in the interval $\left[\tau_{101}-q ; \tau_{101}+q\right]$. This gives rise to a smearing of the signal in $\kappa$ for a given energy transfer, $\varepsilon$.

The dispersion relation for the two modes in bulk hematite is shown in Fig. 4. It is seen that the steepness of the dispersions implies that the related smearing of the scattering vector at low energies is negligible in comparison with the finite-size broadening of the signal of $\delta \kappa \approx 2 \pi / d$ $\approx 0.04 \AA^{-1}$. A bulk-like dispersion would thus have given rise to a signal at $\kappa \approx \tau_{101}$ with a large asymmetric tail towards the high energy side. Since we observe a symmetric peak with essentially no intensity above the two $q=0$ modes, we conclude that the bulk-like continuum is absent and that the spin wave spectrum is quantized, as expected.

\section{B. Properties of the high-energy collective mode in zero field}

The spin structure of bulk hematite is determined by Heisenberg and Dzyaloshinskii exchange interactions and a crystal field anisotropy

$$
\mathcal{H}_{\mathrm{an}}=-\sum_{i}\left(\kappa_{1} S_{i}^{x 2}+\kappa_{\mathrm{Bu}} S_{i}^{22}\right),
$$

where the $x$ direction is along [001] and the $z$ direction is the easy axis within the (001) plane. The coefficients $\kappa_{1}$ and $\kappa_{\mathrm{Bu}}$ represent the anisotropy per atomic site and are related to the anisotropy constants $K_{1}<0$ of the [001] direction and $K_{\mathrm{Bu}}$ $>0$ of the uniaxial basal plane anisotropy defined for hematite nanoparticles in Ref. 13 by $N \kappa_{1} S^{2}=K_{1} V$ and $N \kappa_{\mathrm{Bu}} S^{2}$ $=K_{\mathrm{Bu}} V$, where $S=\frac{5}{2}$ for $\mathrm{Fe}^{3+}, V$ is the particle volume, and $N$ the number of spins. Simplifying the magnetic structure of hematite to a two-sublattice structure, and neglecting the small canting, the zero-field spin wave dispersion relation for wave vector $\boldsymbol{q}$ along the [001] direction is given by ${ }^{21}$

$$
\varepsilon_{q}^{ \pm}=2 \frac{\left\langle S^{z}\right\rangle}{S} \sqrt{\left(\mathcal{A}+\mathcal{J}_{0} \pm \mathcal{J}_{q}\right)\left(\mathcal{B}+\mathcal{J}_{0} \mp \mathcal{J}_{q}\right)},
$$

where $\mathcal{A}=\left(-\kappa_{1}+\kappa_{\mathrm{Bu}}\right) s^{\prime}$ and $\mathcal{B}=\kappa_{\mathrm{Bu}} s^{\prime}$ with $s^{\prime}=S-\frac{1}{2} .{ }^{28}$ Further, $\mathcal{J}_{q}=-S\left(J_{1}+3 J_{3}+6 J_{4}\right) \cos (c q)$, and $\mathcal{J}_{0}=\mathcal{J}_{q=0}$ with $c$ being the lattice constant in the [001] direction. The nearestneighbor out-of-plane exchange constants $J_{n}\left(J_{1}=6 \mathrm{~K}, J_{3}=\right.$ $-29.7 \mathrm{~K}, J_{4}=-23.2 \mathrm{~K}$, which are labeled according to increasing interionic distance) have been determined by spin wave measurements. ${ }^{20}$ Since $J_{0}=556 \mathrm{~K}$ is about 4 orders of magnitude larger than $\mathcal{A}$ and $\mathcal{B}$, the two energy gaps of the dispersion relation for $q \rightarrow 0$ are well approximated by

$$
\varepsilon_{0}^{+} \approx \frac{2\left\langle S^{z}\right\rangle}{S} \sqrt{2 \mathcal{J}_{0} \mathcal{B}} \text { and } \varepsilon_{0}^{-} \approx \frac{2\left\langle S^{z}\right\rangle}{S} \sqrt{2 \mathcal{J}_{0} \mathcal{A}} .
$$

The two branches $( \pm)$ of the dispersion relation are the lowfrequency and high-frequency modes described above. Hence, the two energy gaps (of the two modes at $q=0$ ) correspond to the low-frequency and high-frequency collective magnetic excitation, respectively. Using $\varepsilon_{0}^{+}=0.26 \mathrm{meV}$ and $\varepsilon_{0}^{-}=1.1 \mathrm{meV}$, we find $\mathcal{A}=35 \mathrm{mK}$ and $\mathcal{B}=2.5 \mathrm{mK}$.

In bulk hematite, both modes are of antiferromagnetic character, i.e., the two sublattices precess in antiphase. Due to the strong planar anisotropy, the spin precession is strongly elliptical and very different for the two modes. For the $\varepsilon_{0}^{+}$mode the precession is almost exclusively in the (001) plane, whereas for the $\varepsilon_{0}^{-}$mode it is almost exclusively along the [001] direction. For extreme elliptical precession, the sum in (2) should be replaced by the factor $\left[1-\left(\hat{\boldsymbol{\kappa}} \cdot \hat{\boldsymbol{M}}_{\beta}\right)^{2}\right]$, where $\boldsymbol{M}_{\beta}$ is now along the long axis of the ellipse. This implies that the neutron scattering intensity of the $\varepsilon_{0}^{-}$mode is strongly suppressed when the scattering vector equals $\boldsymbol{\tau}_{003}$, while the $\varepsilon_{0}^{+}$mode has maximum intensity for this scattering vector. In the nanoparticles, we observe exactly this: $I^{-}(003) / I^{-}(101) \approx 0.11$, proving that the high-energy mode is strongly elliptical and predominantly out-of-plane.

In zero applied field we assume that the variation of $\varepsilon_{0}^{-}$ with temperature is solely due to changes in the anisotropy coefficient $\kappa_{1}$. We have no model for this temperature dependence, but as a starting point we fit $\varepsilon_{0}^{-}$to a second-order polynomium in $T$, as shown in Fig. 2(b). The intensity of the mode follows directly from Eq. (2); see the fit in Fig. 2(a). From the definitions of $\mathcal{A}$ and $\mathcal{B}$ we obtain $\mathcal{A}-\mathcal{B}=-\kappa_{1} s^{\prime}$. Further, Eq. (5) relates $\mathcal{A}$ and $\mathcal{B}$ to the energies $\varepsilon_{0}^{-}$and $\varepsilon_{0}^{+}$. 


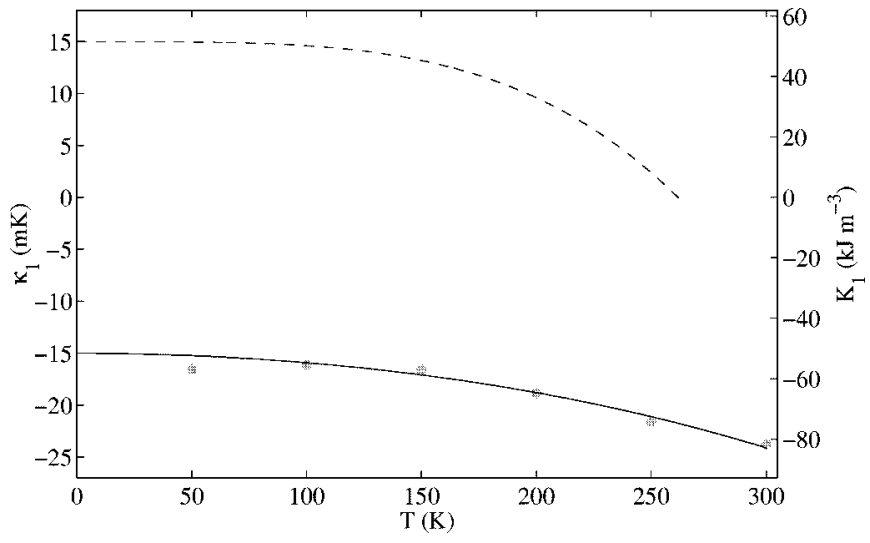

FIG. 5. Temperature dependence of $\kappa_{1}\left(K_{1}\right)$. The solid line represents our results for the hematite nanoparticles. The dashed line represents the value for bulk hematite (Ref. 8).

We have used $\varepsilon_{0}^{+}$as determined in Ref. 13. The obtained value of $\kappa_{1}\left(K_{1}\right)$ is shown in Fig. 5. The value corresponds to $\kappa_{1}=-16(1) \mathrm{mK}$ at $T=0$ and $-23.7(1) \mathrm{mK}$ at room temperature, and hence does not change sign with increasing temperature. This is in contrast to the bulk behavior, where $\kappa_{1}$ change sign at the Morin transition. ${ }^{8}$ Thus, the measured temperature dependence of $K_{1}$ sheds new light on the suppression of the Morin transition in hematite nanoparticles. The overall temperature dependence of the anisotropy in nanoparticles is similar to that of the bulk, but shifted by a constant (positive) amount. In studies of the anisotropy of nanoparticles of $\alpha-\mathrm{Fe},{ }^{23} \quad \gamma-\mathrm{Fe}_{2} \mathrm{O}_{3}$ (maghemite), ${ }^{22}$ and $\alpha-\mathrm{Fe}_{2} \mathrm{O}_{3}$ (hematite, the in-plane anisotropy), ${ }^{24}$ it has been found that the anisotropy constant increases with decreasing particle size. A main contribution to this is presumably surface anisotropy. ${ }^{25}$ It is likely that the different values of $K_{1}$ in bulk and nanoparticles of $\alpha-\mathrm{Fe}_{2} \mathrm{O}_{3}$ also can be explained by the influence of surface anisotropy in nanoparticles.

\section{Effect of an applied field}

The field dependence of the excitation energy is given by ${ }^{8}$

$$
\varepsilon_{0, \boldsymbol{B}}^{ \pm}=\sqrt{\left(\varepsilon_{0}^{ \pm}\right)^{2}+\left(g \mu_{B}\right)^{2}\left[B^{2} \cos ^{2} \xi+B_{D}\left(B \sin \xi+B_{D}\right)\right]}
$$

where $B_{D}$ is the Dzyaloshinsky field and $\xi$ the angle between the applied field $\boldsymbol{B}$ and the [001] direction. Note that due to the small canting, Eq. (6) differs in zero field from Eq. (4) by the term $\left(g \mu_{B} B_{D}\right)^{2}$, which equals $(0.24 \mathrm{meV})^{2}$. Here, we have used the values for bulk hematite ${ }^{8} B_{D}=2.1 \mathrm{~T}$, and $g=2$.

In applied fields, $\varepsilon_{0, \boldsymbol{B}}^{-}$depends on the angle $\xi$ between $\boldsymbol{B}$, which is applied vertically, and the [001] direction. In order to contribute to the scattering, the particles of the powder sample have to be oriented so that their [101] direction coincides with the scattering vector, i.e., $\boldsymbol{\kappa}=\boldsymbol{\tau}_{101}$. Thus, the only degree of freedom is rotation of the (101) plane. Hence, for the different contributing particles the [003] direction will lie on a cone with symmetry axis equal to the [101] direction with varying $\xi$, as illustrated in Fig. 6 . We assume that the contributing particles are randomly oriented and perform an

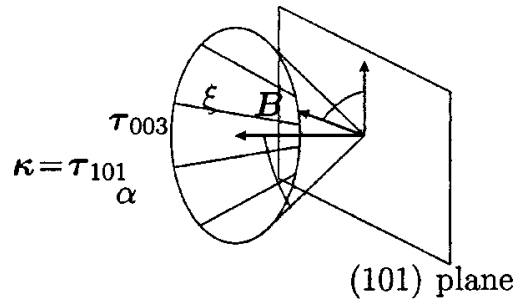

FIG. 6. Illustration of the scattering geometry for a powder sample when $\boldsymbol{\kappa}=\boldsymbol{\tau}_{(101)}$. The [001] direction makes an angle, $\alpha \sim 72^{\circ}$, to the [101] direction.

average of the energy in Eq. (6). In Fig. 2(d) the result is shown for a fit with the bulk values ${ }^{8} B_{D}=2.1 \mathrm{~T}$, and $g=2$. The only free parameter of the fit is the energy at $B=0 \mathrm{~T}$. We obtain $\varepsilon_{0}^{-}=1.12(1) \mathrm{meV}$ at $T=200 \mathrm{~K}$.

The scattered intensity varies for the different contributing particles due to the factor $1-(\hat{\boldsymbol{\kappa}} \cdot \hat{\boldsymbol{M}})^{2}$ in Eq. (2). Again, we perform an average over the contributing particles. We use $\hat{\boldsymbol{M}} \propto\left(-\left[B \sin \xi+B_{D}\right], B \cos \xi, 0\right)$, where the $x$ axis points in the [001] direction and the $z$ axis is perpendicular to the applied field. Hence, we assume a zero-field spin direction given by the projection of the externally applied field onto the basal plane. In Fig. 2(c) the result of the described averaging over Eq. (2) is shown by the solid line. The dotted line models the scattered intensity if we assume approximately circular precessions (with $1-(\hat{\boldsymbol{\kappa}} \cdot \hat{\boldsymbol{M}})^{2}$ in Eq. (2) replaced ${ }^{17,19}$ by $\left.1+(\hat{\boldsymbol{\kappa}} \cdot \hat{\boldsymbol{M}})^{2}\right)$, giving a clearly inferior fit. Hence, these data support the picture of the precession as strongly elliptical. ${ }^{29}$ The strongest evidence for elliptical precession in the highenergy mode, however, comes from the strong suppression of its intensity at $\tau_{003}$.

\section{CONCLUSION}

We have observed a second collective magnetic excitation, the high-frequency mode, in hematite nanoparticles by inelastic neutron scattering at momentum transfers equal to the antiferromagnetic (101) reflection. The precession is found to be strongly anisotropic with a large out-of-plane component. The mode has no dispersion, as seen from the sharpness in energy, giving evidence for quantization of spin waves in the system. A model including an analysis of the orientation of the contributing particles explains the temperature- and field dependence of peak position and scattered intensity. We find that the absolute value of the out-ofplane anisotropy constant $\kappa_{1}$ increases with temperature, without a change of sign as is seen in the bulk material. This is an important step towards explaining the suppression of the Morin transition in hematite nanoparticles.

\section{ACKNOWLEDGMENTS}

We thank F. Bødker for preparing the hematite nanoparticles. We are grateful to B. Lebech and N. B. Christensen for stimulating discussions. This work was supported by the 
Danish Technical Research Council through the framework program on nanomagnetism, and the Danish Natural Science Research Council through the Danish Neutron Scattering
Centre, DANSCATT. This work is based on experiments performed at the SINQ neutron source at the Paul Scherrer Institute, Villigen, Switzerland.
*Electronic address: stine.nyborg.klausen@ risoe.dk

†Electronic address: kim.lefmann@risoe.dk

${ }^{1}$ P. V. Hendriksen, S. Linderoth, and P.-A. Lindgård, Phys. Rev. B 48, 7259 (1993).

${ }^{2}$ C. Mathieu, J. Jorzick, A. Frank, S. O. Demokritov, A. N. Slavin, B. Hillebrands, B. Bartenlian, C. Chappert, D. Decanini, F. Rousseaux, and E. Cambril, Phys. Rev. Lett. 81, 3968 (1998).

${ }^{3}$ J. Jorzick, S. O. Demokritov, C. Mathieu, B. Hillebrands, B. Bartenlian, C. Chappert, F. Rousseaux, and A. N. Slavin, Phys. Rev. B 60, 15194 (1999).

${ }^{4}$ T. M. Crawford, M. Covington, and G. J. Parker, Phys. Rev. B 67, 024411 (2003).

${ }^{5}$ M. Hennion, L. Pardi, I. Mirebeau, E. Suard, R. Sessoli, and A. Caneschi, Phys. Rev. B 56, 8819 (1997).

${ }^{6}$ I. Mirebeau, M. Hennion, H. Casalta, H. Andres, H. U. Güdel, A. V. Irodova, and A. Caneschi, Phys. Rev. Lett. 83, 628 (1999).

${ }^{7}$ R. Caciuffo, G. Amoretti, A. Murani, R. Sessoli, A. Caneschi, and D. Gatteschi, Phys. Rev. Lett. 81, 4744 (1998).

${ }^{8}$ A. H. Morrish, Canted Antiferromagnetism: Hematite (World Scientific, Singapore, 1994).

${ }^{9}$ M. F. Hansen, F. Bødker, S. Mørup, K. Lefmann, K. N. Clausen, and P.-A. Lindgård, Phys. Rev. Lett. 79, 4910 (1997).

${ }^{10}$ F. Bødker, M. F. Hansen, C. B. Koch, K. Lefmann, and S. Mørup, Phys. Rev. B 61, 6826 (2000).

${ }^{11}$ D. Schroeer and R. C. Nininger Phys. Rev. Lett. 19, 632 (1967).

${ }^{12}$ W. Kündig, H. Bömmel, G. Constabaris, and R. H. Lindquist Phys. Rev. 142, 327 (1966).

${ }^{13}$ S. N. Klausen, K. Lefmann, P.-A. Lindgård, K. N. Clausen, M. F. Hansen, F. Bødker, S. Mørup, and M. Telling, J. Magn. Magn. Mater. 266, 68 (2003).

${ }^{14}$ P. R. Christensen, J. L. Bandfield, R. N. Clark, K. S. Edgett, V. E. Hamilton, T. Hoefen, H. H. Kieffer, P. O. Kuzmin, M. D. Lane, M. C. Malin, R. V. Morris, J. C. Pearl, R. Pearson, T. L. Roush, S. W. Ruff, and M. D. Smith, J. Geophys. Res., [Planets] 105, 9623 (2000).

${ }^{15}$ K. Lefmann, D. F. McMorrow, H. M. Rønnow, K. Nielsen, K. N.
Clausen, B. Lake, and G. Aeppli, Physica B 283, 343 (2000).

${ }^{16}$ C. R. H. Bahl, P. Andersen, S. N. Klausen, and K. Lefmann, Nucl. Instrum. Methods Phys. Res. B (to be published in 2004).

${ }^{17}$ P.-A. Lindgård, in Spin Waves and Magnetic Excitations, edited by V. M. Agranovich and A. A. Maradudin, Modern Problems in Condensed Matter Sciences, Vol. 22.1 (North-Holland, Amsterdam, 1988).

${ }^{18}$ C. R. H. Bahl et al. (unpublished).

${ }^{19} \mathrm{~S}$. W. Lovesey, Theory of Neutron Scattering from Condensed Matter (Oxford University Press, Oxford, 1984).

${ }^{20}$ E. J. Samuelsen and G. Shirane, Phys. Status Solidi 42, 241 (1970).

${ }^{21}$ P.-A. Lindgård, A. Kowalska, and P. Laut, J. Phys. Chem. Solids 28, 1357 (1967).

${ }^{22}$ E. Tronc, Nuovo Cimento D 18, 163 (1996).

${ }^{23}$ F. Bødker, S. Mørup, and S. Linderoth, Phys. Rev. Lett. 72, 282 (1994).

${ }^{24}$ F. Bødker and S. Mørup, Europhys. Lett. 52, 217 (2000).

${ }^{25}$ L. Néel, J. Phys. Radium 15, 225 (1954).

${ }^{26}$ M. Nielsen, H. Bjerrum-Møller, P.-A. Lindgård, and A. R. Mackintosh, Phys. Rev. Lett. 25, 1451 (1970).

${ }^{27}$ S. N. Klausen, Magnetic Dynamics of Hematite Nanoparticles (Ph.D. thesis, Department of Physics, Technical University of Denmark, Denmark, 2004).

${ }^{28}$ Eq. (4) differs slightly from that given by Ref. 8, in which a hexagonal anisotropy is included. This cannot be present in a quantum-mechanical derivation for $S=\frac{5}{2}$. Further, the $q$ dependence is ignored in Ref. 8. Magneto-elastic effects could be included in Eq. (4) via $\mathcal{A}$ and $\mathcal{B}$ directly from Ref. 26 . They are effectively included in $\kappa_{B}$.

${ }^{29}$ The described analysis can be applied to all inelastic studies of powder samples; see Ref. 27. In our previous work on the lowfrequency collective magnetic excitations of hematite we did not perform this detailed analysis (Refs. 9 and 13) since there is no angular dependence at $\tau_{003}$; see Ref. 27. 